\title{
Liver disease and mortality among patients with hip fracture: a population-based cohort study
}

This article was published in the following Dove Press journal:

Clinical Epidemiology

\section{Jonathan Montomoli \\ Rune Erichsen \\ Henrik Gammelager \\ Alma B Pedersen}

Department of Clinical Epidemiology, Aarhus University Hospital, Aarhus N, Denmark
Correspondence: Jonathan Montomoli Department of Clinical Epidemiology, Aarhus University Hospital, Olof Palmes Allé 43-45, 8200 Aarhus N, Denmark

Tel +4587167212

Fax +4587167215

Email jonathan.montomoli@gmail.com
Purpose: The aim of this study was to examine the prognostic impact of liver disease on mortality following hip fracture (HF).

Patients and methods: This nationwide cohort study, based on prospectively collected data retrieved from Danish registries, included all patients diagnosed with incident HF in Denmark during 1996-2013. Patients were classified based on the coexisting liver disease at the time of $\mathrm{HF}$, ie, no liver disease, noncirrhotic liver disease, and liver cirrhosis. We computed 30-day and 31-365-day mortality risks. To compare patients with and without liver disease, we computed mortality adjusted hazard ratios (HRs) and corresponding $95 \%$ confidence intervals (CIs) using Cox regression controlled for potential confounders.

Results: Among 152,180 HF patients, 2,552 (1.7\%) patients had noncirrhotic liver disease and $1,866(1.2 \%)$ patients had liver cirrhosis. Thirty-day mortality was $9.4 \%$ among patients with noncirrhotic liver disease, $12.6 \%$ among patients with liver cirrhosis patients, and $9.7 \%$ among patients without liver disease. Compared to patients without liver disease, crude and adjusted HRs within 30 days following HF were, respectively, 0.96 (95\% CI: 0.85-1.10) and 1.24 (95\% CI: 1.09-1.41) for patients with noncirrhotic liver disease and 1.30 (95\% CI: 1.14-1.48) and 2.25 (95\% CI: 1.96-2.59) for those with liver cirrhosis. Among patients who survived 30 days post-HF, the 31-365-day mortality was $18.5 \%$ among patients with noncirrhotic liver disease, $26.4 \%$ among patients with liver cirrhosis, and 19.4\% among patients without liver disease. Corresponding crude and adjusted HRs were, respectively, 0.95 (95\% CI: 0.86-1.04) and 1.08 (95\% CI: 0.99-1.20) for patients with noncirrhotic liver disease and 1.40 (95\% CI: $1.27-1.54)$ and 1.91 (95\% CI: 1.72-2.12) for those with liver cirrhosis.

Conclusion: Liver disease patients, especially those with liver cirrhosis, had increased 30-day mortality and 31-365-day mortality following HF, compared to patients without liver disease. Keywords: epidemiology, liver cirrhosis, hip fracture, mortality, noncirrhotic liver disease

\section{Introduction}

The prevalence of liver disease is increasing worldwide. The end stage of chronic liver disease is liver cirrhosis., ${ }^{1,2}$ Patients with liver disease are prone to develop osteoporosis through mechanisms ranging from osteoclast-mediated bone resorption to bilirubin-related inhibition of osteoblast proliferation. ${ }^{3}$ Moreover, related conditions such as hepatic encephalopathy, alcoholic intoxication, and neuropathy alter proprioception and may increase the risk of falls. ${ }^{4}$ Consequently, patients with liver disease have an increased risk of fracture including hip fracture (HF). ${ }^{3} \mathrm{HF}$ incidence varies across geographic regions, and Denmark has the highest incidence worldwide (574 
cases/100,000 population in 2010). ${ }^{5} \mathrm{HF}$ is considered the most serious osteoporotic fracture, accounting for the majority of fracture-related mortality over the age of 50 years. ${ }^{6} \mathrm{~A}$ recent study showed that the HF risk was fivefold to eightfold increased among patients with alcoholic liver cirrhosis, as compared to the general population of England and Denmark, respectively. ${ }^{7}$

Among patients experiencing HF, 30-day mortality is two times higher than in the general population (matched by age and gender) without fracture ${ }^{8}$ and it has been estimated to be as high as $8-10 \% .{ }^{9,10}$ Mortality risk decreases during the first 6 months postfracture and then stabilizes at $25 \%$ increased risk. ${ }^{8}$

Evidence regarding the specific impact of liver diseases is mostly limited to studies that examined prognostic role of liver disease among several other comorbidities. ${ }^{7,8,11-14}$ Mortality risk among HF patients with liver disease compared to those without liver disease was twofold to fivefold increased for in-hospital ${ }^{11,13,14}$ and 30-day ${ }^{7}$ mortality and nearly twofold increased for 1 year ${ }^{12}$ and overall mortality. ${ }^{8}$ No previous studies have specifically examined the impact of noncirrhotic liver disease and liver cirrhosis on mortality in the first and subsequent months following HF in a population-based setting. New findings regarding the prognosis of patients with liver disease who suffer HF are needed for clinical decision making and individual patient clinical care. We therefore conducted a nationwide cohort study to examine the prognostic impact of liver disease, classified as noncirrhotic liver disease and liver cirrhosis, on the 30-day mortality and the 31-365-day mortality among Danish HF patients.

\section{Patients and methods}

\section{Setting}

We conducted this nationwide population-based cohort study in Denmark (population $=5.6$ million persons during the study period). The Danish National Health Service provides tax-financed medical care to all residents, guaranteeing free and equal access to primary health care and secondary health care. A unique civil personal registration number has been assigned since 1968 to every Danish resident at birth or upon immigration, allowing unambiguous record linkage at the individual level among all Danish registries. ${ }^{15}$

\section{Study population}

The Danish National Patient Registry (DNPR) has recorded all nonpsychiatric hospital admissions since 1977 and all hospital outpatient and emergency contacts since 1995, using the International Classification of Diseases (ICD), eighth revision (ICD-8) until 1993 and tenth revision (ICD-10) thereafter. We identified all patients coded in the DNPR with a first-time HF from January 1, 1996, to December 31, 2013.

HFs were classified as cervical, pertrochanteric, and subtrochanteric fractures. The date of surgery was retrieved for patients who underwent HF surgery. ${ }^{16}$ Patients with pathological and nonpathological HFs as well as patients with isolated HF and those with other traumatic injuries were included in the study population since we did not have data to differentiate those patients. To restrict the study to patients with first-time HF, we excluded those with a diagnosis of HF up to 15 years prior to the index hospitalization/hospital contact qualifying a patient for study inclusion. Moreover, patients with a history of liver transplantation were excluded as they are at increased risk of osteoporosis $(n=11) .{ }^{17}$

\section{Liver disease}

We used the DNPR to identify all diagnoses of liver disease (refer Supplementary materials for diagnostic codes) prior to or on the date of a first-time hospitalization for HF. Patients with a code for liver disease in the DNPR at any time before or during their HF admission were assigned accordingly either to a cohort of patients with noncirrhotic liver disease or to a cohort of patients with liver cirrhosis. Since liver cirrhosis is the end stage of liver disease and is irreversible, in case a patient received diagnoses of both liver cirrhosis and non-cirrhotic liver disease prior to or on the date of a firsttime hospitalization for HF, the patient was assigned to the liver cirrhosis cohort. Patients with no code for liver disease registered in the DNPR before or at the time of their HF were assigned to the third cohort of patients with no liver disease.

\section{Comorbidity}

For each member of our study population, we obtained information from the DNPR on comorbidities registered up to 10 years prior to the incident HF. We used Charlson Comorbidity Index (CCI) scores $^{18}$ to classify patients into the following three comorbidity levels: low (CCI score $=0$ ); moderate (CCI score $=1-2$ ), and high (CCI score $\geq 3$ ). ${ }^{19}$ In addition, we searched the DNPR for previous or concurrent diagnoses of atrial fibrillation or flutter, ${ }^{20,21}$ osteoporosis, ${ }^{3}$ and alcohol-related disease (refer Supplementary materials for diagnosis codes).

\section{Mortality}

We followed patients from the day of hospital admission for HF until death, emigration, or study end (December 31, 2014), whichever occurred first. The Danish Civil Registration System (CRS) registers vital status and residency 
status for all Danish residents. ${ }^{15}$ Vital status records are updated electronically on a daily basis and are virtually complete.

\section{Statistical analysis}

We used the Kaplan-Meier method to compute cumulative 30-day and 31-365-day mortality rates, with 95\% confidence intervals (CIs), for HF patients with and without liver disease. For each cohort, we also computed cumulative 30-day mortality stratified by gender, age group, HF type, comorbidity score, alcohol-related disease (yes/no), osteoporosis (yes/ no), atrial fibrillation (yes/no), and presence/absence of HF surgery within the first 2 days following inpatient admission.

A Cox proportional hazards analysis was used to compute crude hazard ratios and adjusted hazard ratios (aHRs) for the 30-day mortality and the 31-365-day mortality, with 95\% CIs, for patients with liver cirrhosis and noncirrhotic liver disease, compared with patients with no liver disease. In the adjusted Cox model, we controlled for age, gender, CCI score, and history of atrial fibrillation or flutter, osteoporosis, and alcohol-related disease. We also performed a stratified analysis computing hazard ratios for the 30-day mortality, in order to investigate whether the prognostic impact of liver disease varied within subgroups of HF patients. All statistical analyses were performed using the STATA software, Version 12. The study was approved by the Danish Data Protection Board (record no 1-16-02-1-08).

\section{Results Descriptive data}

During the study period, we identified 152,180 HF patients. Among those, 2,552 (1.7\%) patients were previously diagnosed with noncirrhotic liver disease and 1,866 (1.2\%) patients were diagnosed with liver cirrhosis. The remaining 147,762 (97.1\%) patients had no diagnosed liver disease. In our descriptive characterization of the HF cohorts, we found that cervical fracture of the femur was the most frequent HF type, with a prevalence of $57.6 \%$ among patients without liver disease, $52.3 \%$ among those with noncirrhotic liver disease, and 50.4\% among those with liver cirrhosis. Approximately $85 \%$ of patients without liver disease, $84 \%$ of patients with noncirrhotic liver disease, and $82 \%$ of patients with liver cirrhosis underwent hip surgery within 2 days following the hospital admission for HF, increasing to $88-89 \%$ undergoing hip surgery within 7 days following this date irrespective of liver disease. Compared with patients without liver disease, patients with noncirrhotic and cirrhotic liver disease were younger on their HF diagnosis date and were more likely to be male (Table 1). Patients with liver disease were more likely to have chronic pulmonary disease, ulcer disease, diabetes, and alcohol-related disease (Tables 1 and S1). In contrast, atrial fibrillation/flutter was less frequent among HF patients with liver cirrhosis than among patients without liver disease or with noncirrhotic liver disease (Table 1).

\section{Mortality following HF}

Thirty-day mortality was $9.4 \%$ among patients with noncirrhotic liver disease, $12.6 \%$ among patients with liver cirrhosis, and $9.7 \%$ among patients without liver disease (Table 2 and Figure 1). Compared to patients without liver disease, aHRs for 30-day mortality following HF were 1.24 (95\% CI: $1.09-1.41)$ for patients with noncirrhotic liver disease and 2.25 (95\% CI: 1.96-2.59) for patients with liver cirrhosis (Table 2). Absolute 30-day mortality was higher among males than females. Notably, males with liver cirrhosis and those without liver disease had an absolute 30-day mortality of $>13 \%$ while it was $\sim 10 \%$ among males with noncirrhotic liver disease. Absolute 30-day mortality was lower among patients who underwent surgery within the first 2 days following HF than for patients who did not (Table 3). Both in patients with and without liver disease, 30-day mortality increased with age, reaching $\sim 50 \%$ among patients with liver cirrhosis at the age of 90 years or older. Similarly, 30-day mortality increased with higher comorbidity levels among patients without liver disease and those with noncirrhotic liver disease (Table 3). In contrast, among patients with liver cirrhosis, the 30-day mortality was similar in the three subgroups of patients with different comorbidity levels (Table 3 ).

Among patients who were alive 30 days following hospitalization for HF, cumulative mortality after 365 days was similar among patients with noncirrhotic liver disease (18.5\%) and among patients without liver disease (19.4\%) but markedly higher for patients with liver cirrhosis (26.4\%). Compared to patients without liver disease, aHRs for 31-365day mortality were 1.08 ( $95 \%$ CI: $0.99-1.20$ ) for patients with noncirrhotic liver disease and 1.91 (95\% CI: 1.72-2.12) for patients with liver cirrhosis.

In stratified analysis, the negative association between noncirrhotic liver disease and 30-day mortality was not observed for all subgroups of HF patients, but was clear in some subgroups such as females, patients without comorbidities, and patients who underwent surgery (Table 3 ). The impact of liver cirrhosis on 30-day mortality was observed in all patients regardless of patient characteristics. However, aHRs were particularly high in liver cirrhosis patients 
Table I Characteristics of hip fracture patients with and without liver disease, Denmark, 1996-2013

\begin{tabular}{|c|c|c|c|}
\hline Patients' characteristics & $\begin{array}{l}\text { No liver disease, } n(\%) \\
\text { Total }=147,762\end{array}$ & $\begin{array}{l}\text { Noncirrhotic liver disease, } n(\%) \\
\text { Total }=2,552\end{array}$ & $\begin{array}{l}\text { Liver cirrhosis, } \mathrm{n}(\%) \\
\text { Total }=1,866\end{array}$ \\
\hline \multicolumn{4}{|l|}{ Gender } \\
\hline Male & $45,359(30.7 \%)$ & $986(38.6 \%)$ & I,035 (55.5\%) \\
\hline Female & $102,403(69.3 \%)$ & I,566 (6I.4\%) & 831 (44.5\%) \\
\hline \multicolumn{4}{|l|}{ Age (years) } \\
\hline $0-49$ & $5,422(3.7 \%)$ & $202(7.9 \%)$ & $162(8.7 \%)$ \\
\hline $50-59$ & $7,043(4.8 \%)$ & 395 (I5.5\%) & $476(25.5 \%)$ \\
\hline $60-69$ & $15,629(10.6 \%)$ & $521(20.4 \%)$ & $621(33.3 \%)$ \\
\hline $70-79$ & $37,760(25.6 \%)$ & $658(25.8 \%)$ & $416(22.3 \%)$ \\
\hline $80-89$ & 60,486 (40.9\%) & $634(24.8 \%)$ & $174(9.3 \%)$ \\
\hline $90+$ & $21,422(14.5 \%)$ & $142(5.6 \%)$ & $17(0.9 \%)$ \\
\hline \multicolumn{4}{|l|}{ Hip fracture type } \\
\hline Cervical & 85,031 (57.6\%) & I,334 (52.3\%) & 940 (50.4\%) \\
\hline Pertrochanteric & $52,48 \mathrm{I}(35.5 \%)$ & $1,027(40.2 \%)$ & $772(4 \mid .4 \%)$ \\
\hline Subtrochanteric & $10,250(6.9 \%)$ & $191(7.5 \%)$ & 154 (8.3\%) \\
\hline \multicolumn{4}{|l|}{ Comorbidity level ${ }^{a}$} \\
\hline Low & 61,025 (4I.3\%) & $722(28.3 \%)$ & $608(32.6 \%)$ \\
\hline Moderate & $59,063(40.0 \%)$ & $\mathrm{I}, 08 \mathrm{I}(42.4 \%)$ & $817(43.8 \%)$ \\
\hline High & 27,674 (18.7\%) & 749 (29.4\%) & $44 \mathrm{I}(23.6 \%)$ \\
\hline Alcohol-related disease ${ }^{b}$ & $5,133(3.5 \%)$ & $671(26.3 \%)$ & $\mathrm{I}, 077$ (57.7\%) \\
\hline Osteoporosis & $14,343(9.7 \%)$ & $366(14.3 \%)$ & $199(10.7 \%)$ \\
\hline Atrial fibrillation or flutter & 16,718 (11.3\%) & $321(12.6 \%)$ & $143(7.7 \%)$ \\
\hline Hip surgery within 2 days following admission & $125,156(84.7 \%)$ & $2,142(83.9 \%)$ & $\mathrm{I}, 533(82.2 \%)$ \\
\hline
\end{tabular}

Notes: ${ }^{2}$ Calculated according to Charlson Comorbidity Index scores (score =0: no comorbidity; score =I-2: moderate comorbidity; score = 3+: high comorbidity). ${ }^{\text {Including }}$ diagnoses of alcohol abuse and nonhepatic alcohol-related diseases.

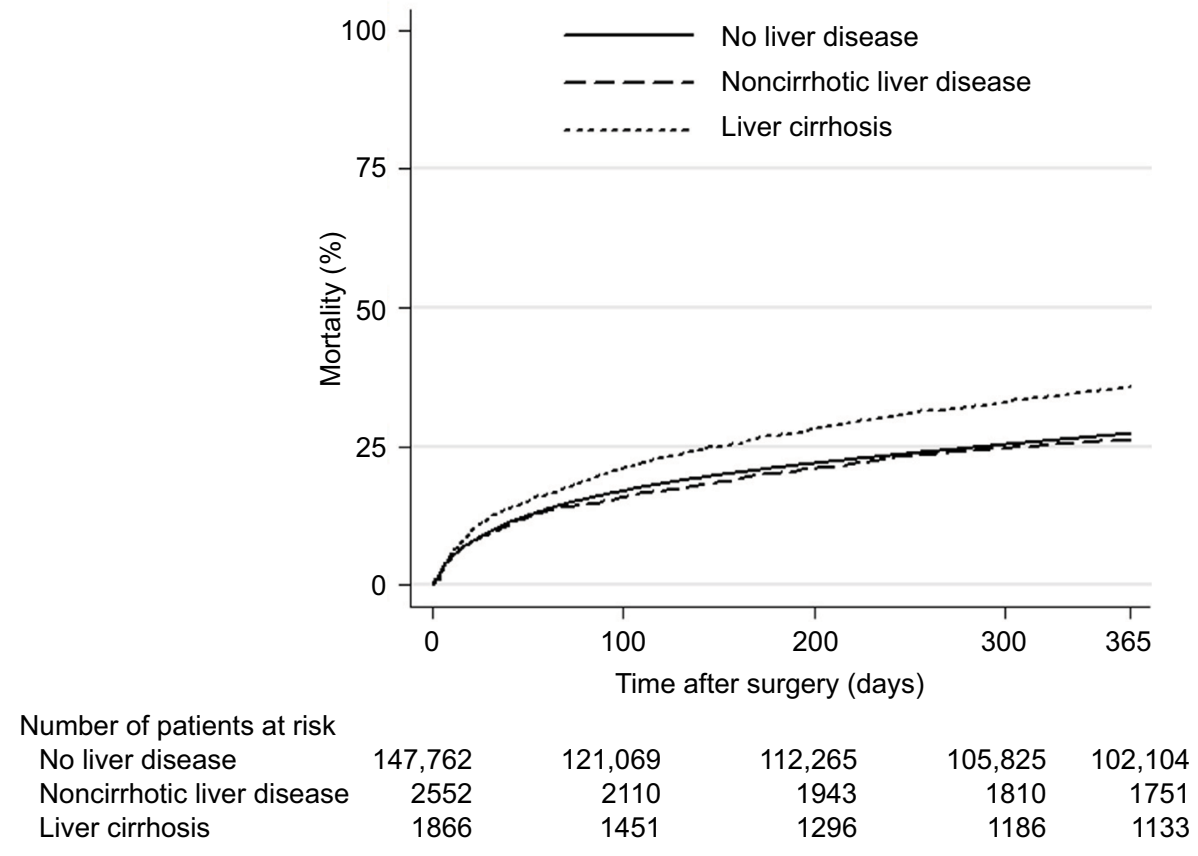

Figure I Cumulative 365-day mortality among hip fracture patients with and without liver disease.

younger than 50 years at the time of HF (aHR 4.92; 95\% CI: Discussion

2.39-10.13) and in those with low comorbidity (aHR 4.47; In this nationwide population-based study, we found that 95\% CI: 3.47-5.76) compared with HF patients without liver disease. liver disease, particularly liver cirrhosis, had a substantial impact on the 30-day mortality and the 31-365-day mortality 
Table 2 Mortality and hazard ratios in patients with and without liver disease within 30 and 31-365 days following hip fracture

\begin{tabular}{|c|c|c|c|c|c|}
\hline \multirow[t]{2}{*}{ Mortality } & \multirow{2}{*}{$\begin{array}{l}\text { Number of } \\
\text { patients (n) }\end{array}$} & \multirow{2}{*}{$\begin{array}{l}\text { Number of } \\
\text { deaths (n) }\end{array}$} & \multirow{2}{*}{$\begin{array}{l}\text { Mortality (\%) } \\
(95 \% \mathrm{Cl})\end{array}$} & \multicolumn{2}{|l|}{ HR (95\% Cl) } \\
\hline & & & & Crude & Adjusted $^{\mathrm{b}}$ \\
\hline \multicolumn{6}{|l|}{ 30-day mortality } \\
\hline No liver disease & 147,762 & $|4,37|$ & $9.7(9.6-9.9)$ & 1.00 & 1.00 \\
\hline Noncirrhotic liver disease & 2,552 & 239 & $9.4(8.3-10.6)$ & $0.96(0.85-1.10)$ & I.24 (I.09-I.4I) \\
\hline Liver cirrhosis & 1,866 & 234 & $12.6(|1.1-| 4.2)$ & $1.30(1.14-1.48)$ & $2.25(1.96-2.59)$ \\
\hline \multicolumn{6}{|l|}{ 31-365-day mortality } \\
\hline No liver disease & 132,824 & 25,379 & $19.4(19.2-19.6)$ & 1.00 & 1.00 \\
\hline Noncirrhotic liver disease & 2,297 & 416 & $18.5(17.0-20.2)$ & $0.95(0.86-1.04)$ & I.08 (0.99-I.20) \\
\hline Liver cirrhosis & 1,623 & 419 & $26.4(24.3-28.6)$ & $1.40(1.27-1.54)$ & $1.91(1.72-2.12)$ \\
\hline
\end{tabular}

Notes: ${ }^{\mathrm{C} C a l c u l a t e d}$ using the Kaplan-Meier method. ${ }^{b}$ Mutually adjusted for gender, age (both as a continuous variable and a categorical variable), comorbidity level, alcoholrelated disease, osteoporosis, and atrial fibrillation or flutter.

Abbreviations: $\mathrm{Cl}$, confidence interval; $\mathrm{HR}$, hazard ratio.

Table 3 Adjusted hazard ratios for 30-day mortality following hip fracture in subgroups of patients with liver disease compared to patients without liver disease

\begin{tabular}{|c|c|c|c|c|c|c|}
\hline \multirow[t]{2}{*}{ Subgroups } & \multicolumn{2}{|l|}{ No liver disease } & \multicolumn{2}{|c|}{ Noncirrhotic liver disease } & \multicolumn{2}{|l|}{ Liver cirrhosis } \\
\hline & $\begin{array}{l}\text { Mortality (\%) } \\
(95 \% \mathrm{CI})\end{array}$ & $\begin{array}{l}\text { Reference } \\
\text { group }\end{array}$ & $\begin{array}{l}\text { Mortality (\%) } \\
(95 \% \mathrm{Cl})\end{array}$ & $\begin{array}{l}\text { Adjusted } \mathbf{H R}^{\mathrm{b}} \\
(95 \% \mathrm{CI})\end{array}$ & $\begin{array}{l}\text { Mortality (\%) } \\
(95 \% \mathrm{Cl})\end{array}$ & $\begin{array}{l}\text { Adjusted } \mathbf{H R}^{\mathrm{b}} \\
(95 \% \mathrm{Cl})\end{array}$ \\
\hline \multicolumn{7}{|l|}{ Gender } \\
\hline Male & $13.4(13.1-13.7)$ & 1.00 & $9.9(8.2-11.9)$ & $1.10(0.90-1.35)$ & $13.8(\mid 1.8-16.0)$ & $2.10(1.76-2.52)$ \\
\hline Female & $8.1(8.0-8.3)$ & 1.00 & $9.1(7.8-10.6)$ & $1.36(1.15-1.61)$ & II.I (9.1-13.4) & $2.58(2.08-3.21)$ \\
\hline \multicolumn{7}{|l|}{ Age (years) } \\
\hline $0-49$ & $0.9(0.6-1.1)$ & 1.00 & $2.0(0.8-5.2)$ & $0.96(0.34-2.75)$ & $8.6(5.2-\mid 4.2)$ & $4.92(2.39-10.13)$ \\
\hline $50-59$ & $2.8(2.4-3.2)$ & 1.00 & $4.3(2.7-6.8)$ & $1.19(0.7 \mathrm{I}-1.99)$ & $9.5(7.2-12.5)$ & $2.57(1.78-3.70)$ \\
\hline $60-69$ & $3.8(3.5-4.2)$ & 1.00 & $6.4(4.6-8.8)$ & $1.39(0.98-1.99)$ & II.8 (9.5-|4.6) & $2.82(2.17-3.68)$ \\
\hline $70-79$ & $6.8(6.6-7.1)$ & 1.00 & $8.1(6.2-10.4)$ & $1.06(0.80-1.39)$ & $15.2(12.1-19.0)$ & $1.83(1.40-2.38)$ \\
\hline $80-89$ & $11.2(11.0-11.5)$ & 1.00 & $14.9(12.3-17.9)$ & $1.29(1.05-1.58)$ & $17.8(12.9-24.4)$ & $1.56(1.09-2.22)$ \\
\hline $90+$ & $19.5(19.0-20.0)$ & 1.00 & $26.8(20.3-34.9)$ & $1.27(0.92-1.75)$ & $47.1(27.0-72.4)$ & $2.19(1.09-4.40)$ \\
\hline \multicolumn{7}{|l|}{ Hip fracture type } \\
\hline Cervical & $9.1(8.9-9.3)$ & 1.00 & $8.8(7.4-10.4)$ & I. $18(0.98-1.42)$ & II.4 (9.5-13.6) & $2.06(1.68-2.52)$ \\
\hline Pertrochanteric & $10.7(10.4-11.0)$ & 1.00 & $9.0(7.4-10.9)$ & $1.39(0.98-1.99)$ & $13.1(10.9-15.7)$ & $2.82(2.17-3.68)$ \\
\hline Subtrochanteric & $10.2(9.6-10.8)$ & 1.00 & $15.6(|| .4-2 \mid .2)$ & $1.06(0.80-1.39)$ & $16.9(\mid 1.8-23.8)$ & $1.83(1.40-2.38)$ \\
\hline \multicolumn{7}{|l|}{ Comorbidity level ${ }^{\mathrm{c}}$} \\
\hline Low & $6.1(5.9-6.3)$ & 1.00 & $6.0(4.5-8.0)$ & $1.68(1.24-2.28)$ & $12.7(10.3-15.6)$ & $4.47(3.47-5.76)$ \\
\hline Moderate & $10.5(10.2-10.7)$ & 1.00 & $8.0(6.5-9.7)$ & $1.12(0.90-1.38)$ & $12.7(10.6-15.2)$ & $2.53(2.05-3.13)$ \\
\hline High & $16.3(15.8-16.7)$ & 1.00 & $14.8(12.4-17.5)$ & $1.17(0.97-1.42)$ & $12.1(9.4-15.5)$ & $1.13(0.85-1.50)$ \\
\hline \multicolumn{7}{|c|}{ Alcohol-related disease $^{d}$} \\
\hline Yes & $6.7(6.0-7.4)$ & 1.00 & $4.6(3.3-6.5)$ & $0.86(0.59-1.25)$ & $10.3(8.6-12.3)$ & $1.81(1.46-2.25)$ \\
\hline No & $9.9(9.7-10.0)$ & 1.00 & II.I (9.7-12.6) & $1.30(1.13-1.49)$ & $15.6(\mid 3.3-18.4)$ & $2.57(2.15-3.07)$ \\
\hline \multicolumn{7}{|l|}{ Osteoporosis } \\
\hline Yes & $9.4(8.9-9.9)$ & 1.00 & $9.1(6.5-12.5)$ & $1.18(0.83-1.67)$ & |4.| (9.9-19.7) & $2.61(1.74-3.93)$ \\
\hline No & $9.8(9.6-9.9)$ & 1.00 & $9.5(8.3-10.8)$ & $1.25(1.09-1.43)$ & $12.4(10.9-14.1)$ & $2.21(1.90-2.56)$ \\
\hline \multicolumn{7}{|c|}{ Atrial fibrillation or flutter } \\
\hline Yes & $17.0(16.5-17.6)$ & 1.00 & $17.6(13.8-22.2)$ & $1.23(0.95-1.61)$ & $15.6(10.5-22.7)$ & $1.28(0.83-1.99)$ \\
\hline No & $8.8(8.7-9.0)$ & 1.00 & $8.2(7.2-9.4)$ & $1.24(1.07-1.44)$ & $12.3(10.9-14.0)$ & $2.45(2.11-2.84)$ \\
\hline \multicolumn{7}{|c|}{ Hip surgery within 2 days } \\
\hline \multicolumn{7}{|l|}{ following admission } \\
\hline Yes & $9.1(9.0-9.3)$ & 1.00 & $8.9(7.8-9.3)$ & $1.27(1.10-1.46)$ & $12.2(10.7-14.0)$ & $2.41(2.06-2.82)$ \\
\hline No & $13.1(12.6-13.5)$ & 1.00 & $12.0(12.6-13.5)$ & $1.15(0.86-1.53)$ & $14.2(\mid 0.8-18.4)$ & $1.71(1.26-2.32)$ \\
\hline
\end{tabular}

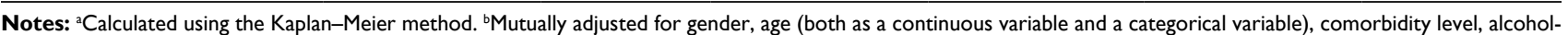
related disease, osteoporosis, and atrial fibrillation or flutter. ${ }^{~ C}$ Calculated according to Charlson Comorbidity Index scores (score $=0$ : no comorbidity; score $=1-2$ : moderate comorbidity; score =3+: high comorbidity). Including diagnoses of alcohol abuse and nonhepatic alcohol-related diseases.

Abbreviations: $\mathrm{Cl}$, confidence interval; $\mathrm{HR}$, hazard ratio. 
following HF. As well, noncirrhotic liver disease was associated with a notably high 30-day mortality risk among HF patients with a low level of comorbidity. Similarly, the relative negative prognostic impact of liver cirrhosis on 30-day mortality was markedly increased among HF patients who had low comorbidity or were aged $<50$ years. Notably, absolute 30-day mortality increased with increasing age among patients with and without liver disease reaching a mortality of $\sim 50 \%$ among patients with liver cirrhosis aged 90 years or older.

To our knowledge, this is the largest population-based cohort study undertaken to date among HF patients to evaluate the prognostic impact of liver disease, adjusting for gender, age, and comorbidity. Our study extends existing knowledge by examining the prognostic impact of liver disease based on its severity.

Although comparison with earlier studies is hampered by differences in definitions of the outcome (ie, in-hospital, 1 year, and overall mortality) and in the identification of patients with liver disease, our findings are on the whole in accordance with those of six earlier studies. ${ }^{7,8,11-14} \mathrm{~A}$ recent study reported absolute 30-day mortality in patients with alcoholic liver cirrhosis compared to general population (matched by age and gender) to be 11.5 vs $5.0 \%$ in England and 10.0 vs $6.6 \%$ in Denmark. ${ }^{7}$ The adjusted mortality risk ratio comparing deaths among patients with alcoholic cirrhosis to controls was 2.8 (95\% CI: $1.9-3.9)$ and 2.0 (95\% CI: 1.5-2.7) for England and Denmark, respectively. ${ }^{7}$ Our findings are supported by previous results. However, mortality was not the primary outcome of the study and stratified analyses were not performed. Moreover, despite similar study period and study population, the lower absolute 30-day mortality in the control Danish cohort compared to our finding was likely due to matching. Two studies reported the impact of liver disease on inpatients' mortality following HF. ${ }^{11,14} \mathrm{~A}$ Spanish study included 31,884 patients older than 65 years with fall-related HF and reported odd ratios for inpatient mortality adjusted for age, gender, and several comorbidities. ${ }^{14}$ Among the 600 patients with chronic liver disease compared to patients without chronic liver disease, the adjusted odds ratio was 2.40 (95\% CI: $1.82-3.17) .{ }^{14}$

Frost et al investigated risk factors for mortality among 1,504 HF patients, including 21 patients with liver disease. They found that liver disease was associated with an adjusted relative risk of 4.75 (95\% CI: 1.95-11.83) for inpatient mortality, controlling for age, gender, and comorbidity. ${ }^{11}$ Two studies have examined the prognostic impact of liver disease (among other comorbidities) on 1-year mortality following
HF. ${ }^{12,13}$ A US study of 45,165 veterans aged $>65$ years found that liver disease was associated with elevated 1-year mortality following HF (aHR $=1.92 ; P<0.0001) .{ }^{12}$ The other study, based on Canadian hospital discharge data for 3,981 HF patients older than 60 years, reported that liver disease was associated with an unadjusted odds ratio of 2.7 (95\% CI: 1.0-7.0) for 1-year mortality. ${ }^{13}$ A 2007 Danish study found that HF patients with liver disease treated during 1977-2001 had $68 \%$ higher mortality than patients without liver disease (median follow-up among HF patients $=3.8$ years). ${ }^{8}$ In this previous study, Vestergaard et $\mathrm{al}^{8}$ assessed the effects of premorbid conditions on the risk of long-term mortality following HF and examined whether causes of death were related to preexisting conditions or to postoperative complications. The study compared HF patients to persons without HF (matched on age and gender, but not on comorbidity) and found that excess mortality after HF was due to complications of the fracture event rather than to preexisting comorbidity. This conclusion was based on the finding that causes of death categorized as trauma related were more frequent among patients with HF than in the general population. Also, among patients with HF compared to general population, unadjusted hazard ratios for mortality did not increase according to the severity of comorbidity in stratified analyses. However, aHRs increased with comorbidity level when HF patients with different degrees of comorbidity were compared with HF patients with no comorbidity. Our findings showed that absolute 30-day mortality remained $\sim 12 \%$ among patients with liver cirrhosis independently from the comorbidity level, while it increased with increasing comorbidity level in both patients with noncirrhotic liver disease and in patients without liver disease. Corresponding aHRs for 30-day mortality for patients with liver cirrhosis compared to patients without liver disease were markedly higher for patients with low comorbidity level than those with high comorbidity level mainly due to changes in absolute mortality in patients without liver disease. Similarly, the higher impact of liver disease among patients undergoing HF surgery or among patients younger than 50 years may be explained by lower absolute mortality among patients without liver disease. Indeed, those patients were likely to be less severely ill than patients who did not received surgery or patients older than 50 years.

Other studies have shown that severity of comorbidity burden predicts inpatient complications after hip replacement ${ }^{10,22,23}$ and that patients with liver disease are at increased risk of complications after hip arthroplasty ${ }^{24}$ and other surgery. ${ }^{25}$ Liver disease is multisystemic, and affected patients are at increased risk of venous thromboembolism, hypo- and 
hypercoagulopathy, infections, hemodynamic instability, and malnutrition. ${ }^{26}$ These conditions may lead to life-threatening complications in patients hospitalized with HF. Moreover, impaired bone metabolism is likely to affect HF healing leading to delayed patient mobilization and worsening health condition to such an extent that affects long-term mortality. The strengths of our study are its nationwide populationbased design and completeness of follow-up in a uniform health care system. Moreover, the positive predictive value of the HF diagnosis in the DNPR is expected to be as high as $98 \%$ for the most common femoral neck fractures and $\sim 63 \%$ for subtrochanteric fractures as shown in previous studies on Scandinavian databases. ${ }^{27,28}$

The study may be limited by potential misclassification bias since the extent of liver disease could be defined only roughly, via classification into the following two groups: liver cirrhosis and noncirrhotic liver disease. It is likely that patients with asymptomatic liver disease were underdiagnosed and that an unknown number of patients assigned to the noncirrhotic liver disease cohort also had undiagnosed liver cirrhosis. ${ }^{29}$ This would make our estimates conservative. ${ }^{30}$ The inclusion of both pathological and nonpathological fractures may have biased our estimates in the eventuality of a higher incidence of pathological HFs among patients with liver disease. However, patients with liver disease were not more likely to have a diagnosis of "any tumor" and "metastatic tumor" than patients without liver disease (Table S1). Therefore, we do not believe this would have had a strong impact on our findings. Finally, we lacked information on other measurements of frailty except the comorbidities included in the Charlson comorbidity score that have been reported to have a positive predictive value that range from 85 to $100 \%{ }^{19}$ thus, we cannot rule out residual confounding. These limitations make it difficult to suggest guidelines for the clinical care of patients with liver disease who sustain a HF. It is important, however, for clinicians to consider liver disease as a prognostic indicator of recovery.

\section{Conclusion}

We found that patients with liver disease, especially those with liver cirrhosis, had markedly increased 30-day mortality and 31-365-day mortality after HF, compared with patients without liver disease. Among patients with liver disease, mortality risk following HF was notably high for patients with an otherwise low comorbidity level. Moreover, the prognostic impact of liver cirrhosis was high among patients younger than 50 years.

\section{Acknowledgments}

This study was supported by the Program for Clinical Research Infrastructure (PROCRIN) established by the Lundbeck Foundation and the Novo Nordisk Foundation. The corresponding author had full access to all the data in the study and had final responsibility for the decision to submit for publication. The funding source is not involved in any decision process regarding study design; collection, analysis, and interpretation of data; in the writing of the report; and in the decision to submit the article for publication.

\section{Disclosure}

The authors report no conflicts of interest in this work.

\section{References}

1. Blachier M, Leleu H, Peck-Radosavljevic M, Valla DC, RoudotThoraval F. The burden of liver disease in Europe: a review of available epidemiological data. J Hepatol. 2013;58(3):593-608.

2. Younossi ZM, Stepanova M, Afendy M, et al. Changes in the prevalence of the most common causes of chronic liver diseases in the United States from 1988 to 2008. Clin Gastroenterol Hepatol. 2011;9(6):524-530.

3. Nakchbandi IA. Osteoporosis and fractures in liver disease: relevance, pathogenesis and therapeutic implications. World J Gastroenterol. 2014;20(28):9427-9438.

4. Ezaz G, Murphy SL, Mellinger J, Tapper EB. Increased morbidity and mortality associated with falls among patients with cirrhosis. Am JMed. 2018;131(6):645-650.

5. Kanis JA, Oden A, McCloskey EV, et al. A systematic review of hip fracture incidence and probability of fracture worldwide. Osteoporos Int. 2012;23(9):2239-2256.

6. Johnell O, Kanis JA. An estimate of the worldwide prevalence and disability associated with osteoporotic fractures. Osteoporos Int. 2006;17(12):1726-1733.

7. Otete H, Deleuran T, Fleming KM, et al. Hip fracture risk in patients with alcoholic cirrhosis: a population-based study using English and Danish data. J Hepatol. Epub 2018 Apr 17.

8. Vestergaard P, Rejnmark L, Mosekilde L. Increased mortality in patients with a hip fracture-effect of pre-morbid conditions and post-fracture complications. Osteoporos Int. 2007;18(12):1583-1593.

9. Giannoulis D, Calori GM, Giannoudis PV. Thirty-day mortality after hip fractures: has anything changed? Eur J Orthop Surg Traumatol. 2016;26(4):365-370.

10. Pedersen AB, Ehrenstein V, Szepligeti SK, et al. Thirty-five-year trends in first-time hospitalization for hip fracture, 1-year mortality, and the prognostic impact of comorbidity: a Danish Nationwide Cohort Study, 1980-2014. Epidemiology. 2017;28(6):898-905.

11. Frost SA, Nguyen ND, Black DA, Eisman JA, Nguyen TV. Risk factors for in-hospital post-hip fracture mortality. Bone. 2011;49(3):553-558.

12. Bass E, French DD, Bradham DD, Rubenstein LZ. Risk-adjusted mortality rates of elderly veterans with hip fractures. Ann Epidemiol. 2007;17(7):514-519.

13. Jiang HX, Majumdar SR, Dick DA, et al. Development and initial validation of a risk score for predicting in-hospital and 1-year mortality in patients with hip fractures. J Bone Miner Res. 2005;20(3):494-500.

14. Padron-Monedero A, Lopez-Cuadrado T, Galan I, Martinez-Sanchez EV, Martin P, Fernandez-Cuenca R. Effect of comorbidities on the association between age and hospital mortality after fall-related hip fracture in elderly patients. Osteoporos Int. 2017;28(5):1559-1568.

15. Schmidt M, Pedersen L, Sorensen HT. The Danish Civil Registration System as a tool in epidemiology. Eur J Epidemiol. 2014;29(8): 541-549. 
16. Nyholm AM, Gromov K, Palm H, et al. Time to surgery is associated with thirty-day and ninety-day mortality after proximal femoral fracture: a retrospective observational study on prospectively collected data from the Danish Fracture Database Collaborators. J Bone Joint Surg Am. 2015;97(16):1333-1339.

17. Compston JE. Osteoporosis after liver transplantation. Liver Transpl. 2003;9(4):321-330.

18. Charlson ME, Pompei P, Ales KL, MacKenzie CR. A new method of classifying prognostic comorbidity in longitudinal studies: development and validation. J Chronic Dis. 1987;40(5):373-383.

19. Thygesen SK, Christiansen CF, Christensen S, Lash TL, Sorensen HT. The predictive value of ICD-10 diagnostic coding used to assess Charlson comorbidity index conditions in the population-based Danish National Registry of Patients. BMC Med Res Methodol. 2011;11:83.

20. Adunsky A, Arad M, Koren-Morag N, Fleissig Y, Mizrahi EH. Increased 1-year mortality rates among elderly hip fracture patients with atrial fibrillation. Aging Clin Exp Res. 2012;24(3):233-238.

21. Mozos I. Arrhythmia risk in liver cirrhosis. World J Hepatol. 2015;7(4): $662-672$.

22. Greenfield S, Apolone G, McNeil BJ, Cleary PD. The importance of co-existent disease in the occurrence of postoperative complications and one-year recovery in patients undergoing total hip replacement. Comorbidity and outcomes after hip replacement. Med Care. 1993;31(2):141-154.
23. Glassou EN, Pedersen AB, Hansen TB. Is decreasing mortality in total hip and knee arthroplasty patients dependent on patients' comorbidity? Acta Orthop. 2017;88(3):288-293.

24. Deleuran T, Vilstrup H, Overgaard S, Jepsen P. Cirrhosis patients have increased risk of complications after hip or knee arthroplasty. Acta Orthop. 2014;86(1):108-113.

25. Ziser A, Plevak DJ, Wiesner RH, Rakela J, Offord KP, Brown DL. Morbidity and mortality in cirrhotic patients undergoing anesthesia and surgery. Anesthesiology. 1999;90(1):42-53.

26. Keegan MT, Plevak DJ. Preoperative assessment of the patient with liver disease. Am J Gastroenterol. 2005;100(9):2116-2127.

27. Baron JA, Lu-Yao G, Barrett J, McLerran D, Fisher ES. Internal validation of Medicare claims data. Epidemiology. 1994;5(5):541-544.

28. Hudson M, Avina-Zubieta A, Lacaille D, Bernatsky S, Lix L, Jean S. The validity of administrative data to identify hip fractures is high - a systematic review. J Clin Epidemiol. 2013;66(3):278-285.

29. Almdal TP, Sorensen TI. Incidence of parenchymal liver diseases in Denmark, 1981 to 1985 : analysis of hospitalization registry data. The Danish Association for the Study of the Liver. Hepatology. 1991;13(4): 650-655.

30. Rothman KJ, Greenland S, Lash TL. Validity in epidemiological studies. In: Rothman KJ, Greenland S, Lash TL, editors. Modern Epidemiology. Third ed. Philadelphia: Wolters Kluwer Health/Lippincott Williams \& Wilkins; 2008:138-144. 


\section{Supplementary materials International Classification of Diseases (ICD) codes and surgical codes used in the analysis}

The following codes were used to identify conditions in the Danish National Patient Registry:

- Hip fracture: ICD-8: 820; ICD-10: S72.0, S72.1, S72.2

- Chronic liver disease:

○ Liver cirrhosis: ICD-8: 571.09, 571.90, 571.91, 571.92, 571.99; ICD-10: K70.3, K71.7, K74.3-K74.6

○ Noncirrhotic alcoholic liver disease: ICD-8: 571.10, 571.19; ICD-10: K70.0, K70.1, K70.2, K70.4, K70.9

- Noncirrhotic nonalcoholic liver disease: ICD-8: 70.01-70.09, 570.00-573.09 (excluding 571.10, 571.19, 571.09, 571.90, 571.91, 571.92, 571.99); ICD-10: B15-B19, K71.0-K71.9, K72, K73, K74.0, $\mathrm{K} 74.1, \mathrm{~K} 74.2, \mathrm{~K} 75, \mathrm{~K} 76, \mathrm{~K} 77, \mathrm{R} 74.0$ (excluding K70.3, K71.7, K74.3, K74.4, K.74.5, K74.6)

- Nonalcoholic steatohepatitis and nonalcoholic fatty liver disease: ICD-8: 571.11, 571.19; ICD-10: K75.8, K76.0

- Liver transplantation (exclusion criteria for chronic liver disease cohort): ICD-8 (1977-1995): 47270, 47279; Nordic Medico-Statistical Committee Surgery Codes (1996 to present): KJJC

- Alcohol-related disease other than liver disease: ICD-8: 291-291.9, 303-303.9, 980; ICD-10: F10.2, F10.7, F10.8, I42.6, G62.1, K29.2, G72.1, G31.2, Z72.1, BRHE20, K860, K852

- Diseases included in the modified Charlson Comorbidity Index:

○ Myocardial infarction: ICD-8: 410; ICD-10: I21, I22, $\mathrm{I} 23$

○ Congestive heart failure: ICD-8: 427.09, 427.10, 427.11, 427.19, 428.99, 782.49; ICD-10: I50, I11.0, I13.0, I13.2 o Peripheral vascular disease: ICD-8: 440, 441, 442, 443, 444, 445; ICD-10: I70, I71, I72, I73, I74, I77

○ Cerebrovascular disease: ICD-8: 430-438; ICD-10: I60-I69, G45, G46

○ Dementia: ICD-8: 290.09-290.19, 293.09; ICD-10: F00-F03, F05.1, G30

- Chronic pulmonary disease: ICD-8: 490-493, 515518; ICD-10: J40-J47, J60-J67, J68.4, J70.1, J70.3, J84.1, J92.0, J96.1, J98.2, J98.3

○ Connective tissue disease: ICD-8: 712, 716, 734, 446, 135.99; ICD-10: M05, M06, M08, M09, M30, M31, M32, M33, M34, M35, M36, D86

○ Ulcer disease: ICD-8: 530.91, 530.98, 531-534; ICD10: K22.1, K25-K28

- Uncomplicated type 1 and type 2 diabetes: ICD-8: 249.00, 249.06, 249.07, 249.09, 250.00, 250.06, 250.07, 250.09; ICD-10: E10.0, E10.1, E10.9, E11.0, E11.1, E11.9

○ Hemiplegia: ICD-8: 344; ICD-10: G81, G82

○ Moderate-to-severe renal disease: ICD-8: 403, 404, 580-583, 584, 590.09, 593.19, 753.10-753.19, 792; ICD-10: I12, I13, N00-N05, N07, N11, N14, N17N19, Q61

○ Diabetes with end-organ damage: ICD-8: 249.01249.05, 249.08, 250.01-250.05, 250.08; ICD-10: E10.2-E10.8, E11.2-E11.8

○ Any tumor: ICD-8: 140-19; ICD-10: C00-C75

○ Leukemia: ICD-8: 204-207; ICD-10: C91-C95

○ Lymphoma: ICD-8: 200-203, 275.59; ICD-10: C81C85, C88, C90, C96

○ Metastatic solid tumor: ICD-8: 195-198, 199; ICD10: C76-C80

○ AIDS: ICD-8: 079.83; ICD-10: B21-B24

- Osteoporosis: ICD-8: 723.09; ICD-10: M80, M81, M82

- Atrial fibrillation and/or flutter: ICD-8: 427.93, 427.94; ICD-10: I48

- Hip surgery: KNFB or KNFJ 
Table SI Prevalence of individual diseases from the Charlson Comorbidity Index in hip fracture patients with and without liver diseases, Denmark, 1996-2013

\begin{tabular}{|c|c|c|c|}
\hline Individual diseases & $\begin{array}{l}\text { No liver disease, } n(\%) \\
\text { Total }=147,762\end{array}$ & $\begin{array}{l}\text { Noncirrhotic liver disease, } n(\%) \\
\text { Total }=2,552\end{array}$ & $\begin{array}{l}\text { Liver cirrhosis, } \mathbf{n}(\%) \\
\text { Total }=1,866\end{array}$ \\
\hline Myocardial infarction & $10,572(7.2)$ & $182(7.1)$ & $74(4.0)$ \\
\hline Congestive heart failure & $13,730(9.3)$ & $310(12.2)$ & $16 \mid(8.6)$ \\
\hline Peripheral vascular disease & $10,78 \mid(7.3)$ & $281(11.0)$ & $127(6.8)$ \\
\hline Cerebrovascular disease & $27,492(18.6)$ & $526(20.6)$ & $329(17.6)$ \\
\hline Dementia & $10,749(7.3)$ & $187(7.3)$ & $126(6)$. \\
\hline Chronic pulmonary disease & $16,979(11.5)$ & $473(18.5)$ & $317(17.0)$ \\
\hline Connective tissue disease & $7,950(5.4)$ & $184(7.2)$ & $80(4.3)$ \\
\hline Ulcer disease & $12,402(8.4)$ & $432(16.9)$ & $574(25.6)$ \\
\hline Diabetes types I and 2 & II,078 (7.5) & $369(14.5)$ & $334(17.9)$ \\
\hline Hemiplegia & $802(0.5)$ & $14(0.6)$ & $5(0.3)$ \\
\hline Moderate-to-severe renal disease & $4,007(2.7)$ & $162(6.4)$ & $82(4.4)$ \\
\hline Diabetes with end-organ damage & $5,786(3.9)$ & $237(9.3)$ & $198(10.6)$ \\
\hline Any tumor & $22,786(15.4)$ & 400 (I5.7) & $208(11.2)$ \\
\hline Leukemia & $603(0.4)$ & II (0.4) & $3(0.2)$ \\
\hline Lymphoma & $\mathrm{I}, \mathrm{I} I 7(0.8)$ & $26(1.0)$ & $16(0.9)$ \\
\hline Metastatic solid tumor & $2,624(1.8)$ & $46(1.8)$ & $33(1.8)$ \\
\hline AIDS & $44(<0.1)$ & $25(0.9)$ & $5(0.3)$ \\
\hline
\end{tabular}

\section{Publish your work in this journal}

Clinical Epidemiology is an international, peer-reviewed, open access, online journal focusing on disease and drug epidemiology, identification of risk factors and screening procedures to develop optimal preventative initiatives and programs. Specific topics include: diagnosis, prognosis, treatment, screening, prevention, risk factor modification,

Submit your manuscript here: https://www.dovepress.com/clinical-epidemiology-journal systematic reviews, risk and safety of medical interventions, epidemiology and biostatistical methods, and evaluation of guidelines, translational medicine, health policies and economic evaluations. The manuscript management system is completely online and includes a very quick and fair peer-review system, which is all easy to use. 